\title{
Acute flaccid paralysis following spinal anaesthesia: a diagnostic dilemma
}

\author{
AFS Dassanayake* \\ Registrar in Anaesthesiology, National Hospital of Sri Lanka, Sri Lanka. \\ *Corresponding author: asithadassanayake@yahoo.com
}

\begin{abstract}
We report on a patient who developed an acute flaccid paralysis following spinal anaesthesia for a right sided inguinal hernia repair and orchidopexy, while on post exposure prophylaxis with anti-rabies vaccination following a dog bite.
\end{abstract}

Keywords: Acute flaccid paralysis; rabies exposure; spinal anaesthesia

\section{Introduction}

Acute flaccid paralysis is a clinical syndrome characterised by rapid onset of weakness including (less frequently) weakness of muscles of respiration and swallowing progressing to maximum severity within several days to weeks. ${ }^{1}$

Neurological complications such as anterior spinal artery syndrome, transverse myelitis, chronic arachnoiditis, cauda-equina syndrome following neuraxial anaesthesia have been documented in the literature, however complications following well conducted spinal anaesthesia are rare. Though in most cases the exact aetiology cannot be detected, various theories such as direct trauma, chemical irritation and sepsis have been postulated as the probable cause.

\section{Case Report}

A 45 year old ASA 1 male patient presented to a local hospital for an elective right-sided inguinal hernia, mesh repair and orchidopexy. He underwent an uneventful intra-operative period. However, on post-op day 1 he had complained of weakness and numbness of the right leg and he was struggling to walk without aid. Neurological examination revealed a sensory loss up to L2 segment. There were no obvious constitutional symptoms or signs and no evidence of meningism. On the same day he was transferred to the neuro-surgical unit at the National hospital of Sri Lanka for further investigation and management.

On admission to the neuro-surgical unit he had downward plantar reflexes and motor power was less on the right lower limb in comparison to the left. Sensory level kept progressing up to T10 within the next 24 hours and an urgent MRI was done, which revealed inflammation of the spinal cord, brain stem and thalamus. (Figure 1) Spinal haematoma was excluded.

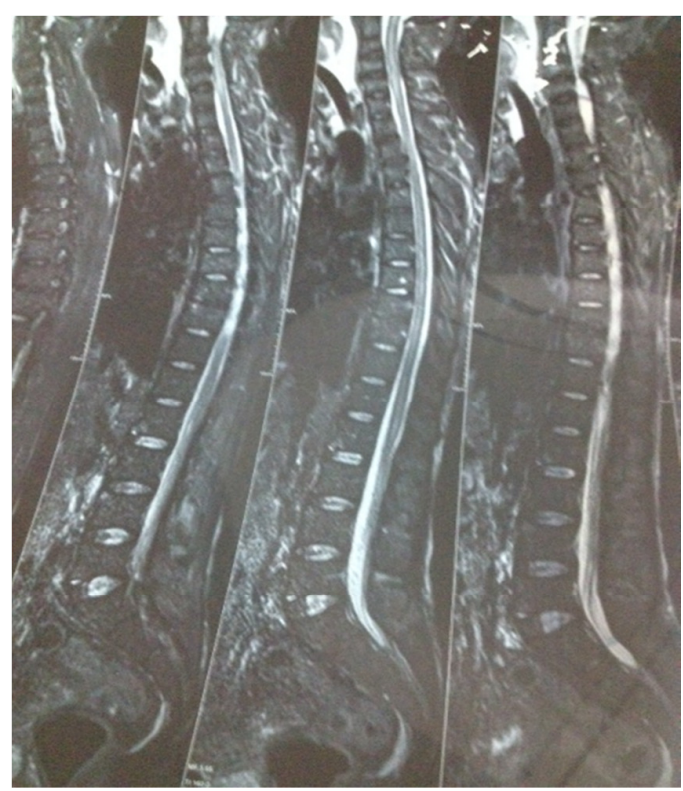

Figure 1.MRI report revealed inflammation of the spinal cord

He became restless while in the ward and subsequently was found to be drowsy. With deteriorating levels in his Glasgow Coma scale (from GCS $15 / 15$ to GCS 10/15) he was admitted to the neuro-intensive care unit for further management. Over the next 2 days he developed an ascending flaccid paralysis and was intubated electively due to deteriorating level of consciousness and respiratory insufficiency.

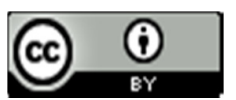

(c) 2016 Dassanayake. This is an Open Access article distributed under the terms of the Creative Commons 
Diagnostic lumbar puncture was done which aspirated clear CSF and examination was normal. CSF culture did not grow any bacteria. Nerve conduction studies revealed motor conduction abnormality probably due to the involvement of the anterior horn cells or root by the same disease process of myelitis. Haematological and biochemical investigations were normal except for an elevated white cell count. PCR and Mantoux test for tuberculosis was negative.

A tracheostomy was performed early to facilitate weaning and removal of secretions. He continued to deteriorate within the next five days despite ventilatory and inotropic support. Antibiotics were commenced empirically. He was treated with intravenous cefuroxime $750 \mathrm{mg}$ 8 hourly along with intravenous acyclovir $500 \mathrm{mg} 8$ hourly during his ICU stay.

A vaccination record was produced by his relative seven days later whereby he was scheduled to receive his fourth and final dose of the anti-rabies vaccine while in ICU, following an unprovoked dog bite by a stray dog 20 days back. This information had not been conveyed to the anaesthetist at the local hospital prior to surgery neither by the patient or his family members. Antibody assay for rabies was positive.

Despite maximum support in ICU he succumbed after 15 days following bulbar and probably respiratory muscle paralysis secondary to what we believe was paralytic rabies.

Post-mortem findings revealed a congested dura with dilated blood vessels and lymphocyte aggregation in peripheral regions of the spinal cord and perivascular cuffing which was in favour of a viral infection. Except for endocardial fibrosis and coronary artery occlusion (confirming an old myocardial infarction) the rest of the organs were unremarkable. Virology studies on the brain confirmed the diagnosis as Rabies.

\section{Discussion}

Rabies is a viral infection affecting the peripheral and central nervous system causing encephalitis or meningo-encephalitis and almost inevitable death. ${ }^{1}$ This fatal disease remains a serious health problem especially in the developing countries.
Surgical intervention in a patient on anti-rabies vaccine is a dilemma as it may cause a reduction in the efficacy of the vaccine through an immunosuppressive effect due to anaesthesia and surgery. This important aspect of history was not known in this patient when he presented for surgery.

The initial presentation of rabies can be diverse and a history of animal contact is rarely obtained. However, rabies should be considered in any patient with rapidly progressing encephalitis. $^{2}$

The clinical presentation of this zoonotic disease is often reported categorically as either encephalitic (furious) or paralytic (dumb), the latter which describes impairment of the spinal cord and peripheral nerve functions. Paralytic rabies is characterised by delayed viral neuro invasion and a more intense inflammation than furious rabies. ${ }^{2}$

Incubation period for rabies is the most variable of all acute CNS infections. ${ }^{3}$ This patient probably presented during the prodromal phase as in paralytic rabies. The local manifestations are the earliest symptoms which perhaps were neglected by the patient. Hallmark of paralytic rabies is an ascending paralysis ${ }^{4}$ and this was evident in our patient.

There are very few reports on the safety and anaesthesia and surgery in a patient receiving anti-rabies vaccination. The key issues of importance are the possible reduction in the efficacy of the vaccine and the possible need to avoid local anaesthetic for post-operative analgesia to assist in the early detection of any neurological deficits. ${ }^{5}$

Apart from a number of reasons contributing to post-exposure prophylaxis failure, ketamine anaesthesia has been mentioned as an additional risk factor for treatment failure. ${ }^{5}$

The main concern in this patient was the probability of reduction in the efficacy of the vaccine due to immunosuppressive effect of anaesthesia and surgery.

Short et al conducted a postal survey regarding anaesthesia and immunization and they recommended that elective surgery and anaesthesia should be postponed for one week after inactive vaccination and for 3 weeks after a 
live attenuated vaccination in children. ${ }^{6}$ On the other hand neuraxial anaesthesia is believed to be associated with modest preservation of the immune function compared to general anaesthesia as it attenuates the endocrine response to surgery. However, the use of regional anaesthesia needs further evaluation in patients receiving anti-rabies treatment. ${ }^{5}$

The use of neuraxial anaesthesia in a patient on rabies post exposure prophylaxis could have medico-legal implications. It is known that rabies may present with a paralytic or "Guillian Barre" like syndrome, without the typical excitation, spontaneous inspiratory spasms and hydrophobia. Any neurological deficit because of the disease per se can be attributed to a regional block or vice versa. This could pose a diagnostic dilemma to the anaesthetist.

A lesson that we could all learn from this case report is that details of any pre or post exposure prophylaxis with regard to immunization already given must be recorded during the pre-operative assessment and it is also mandatory to document and explain the possible consequences to the patient prior to anaesthesia, although it has been reported rarely, that rabies vaccine per se can cause a neurological illness simulating "Guillian Barre" syndrome after post exposure prophylaxis.

\section{References}

1. Marx A, Glass JD, Sutter RW. Differential Diagnosis of Acute Flaccid Paralysis and its role in Poliomyelitis Surveillance, Epidemiol Rev,2000;22(2)

http://dx.doi.org/10.1093/oxfordjournals.epirev.a 018041

PMid:11218380

2. Henderson H, Rupprecht CE. Infectious Disease and Antimicrobial Agents. Rabies virus www.antimicrobe.org

3. Laothamatas J. Fusion and Paralytic rabies of canine origin; neuroimaging with virological and cytokine studies, J Neurovirol, 2008; 14(2):11929

http://dx.doi.org/10.1080/13550280701883857 PMid: 18444083

4. Sudana SNM, Sukumaran SM. Antemortem diagnosis and prevention of human rabies, Annals of Indian Academy of Neurology, 2008; 11(1):3-12

http://dx.doi.org/10.4103/0972-2327.40219 PMid:19966972 PMCid:PMC2781142

5. Rewari V, Garg R, Trikha A. Rabies Vaccine and Neuraxial Anaesthesia, S Afr J
AnaesthsiolAnalg,2010;16(5):32-34 http://dx.doi.org/10.1080/22201173.2010.10872 707

6. Hart J A, Vanderwalt J H, Zoanetti D C. Immunization and Anaesthesia an International Survey, Paediatric Anaesthesia, 2006; 16(2): 514-522 